\title{
Reactive Force Field for Liquid Hydrazoic Acid with Applications to Detonation Chemistry - Supporting Information
}

David Furman ${ }^{1,4}$, Faina Dubnikova ${ }^{1}$, Adri C.T. van Duin $^{2}$, Yehuda Zeiri ${ }^{3,4}$ and Ronnie Kosloff ${ }^{1}$

${ }^{1}$ Fritz Haber Research Center for Molecular Dynamics, Institute of Chemistry, Hebrew University of Jerusalem, Jerusalem 91904, Israel

${ }^{2}$ Department of Mechanical and Nuclear Engineering, Pennsylvania State University, University Park, Pennsylvania 16802, USA

3Biomedical Engineering, Ben Gurion University, Beer-Sheva 94105, Israel

4Division of Chemistry, NRCN, P.O. Box 9001, Beer-Sheva 84190, Israel

Reactive MD-force field: nitramines (RDX/HMX/TATB/PETN))+DMNA-barrier+innervdWaa+HN3 Furman et al., (2015)

39 ! Number of general parameters

48.3758 !Overcoordination parameter

6.1580 !Overcoordination parameter

29.5996 !Valency angle conjugation parameter

216.5421 !Triple bond stabilisation parameter

12.2245 !Triple bond stabilisation parameter

0.0000 !C2-correction

1.0701 !Undercoordination parameter

7.5000 !Triple bond stabilisation parameter

10.5720 !Undercoordination parameter

8.8937 !Undercoordination parameter

-17.7310 !Triple bond stabilization energy

0.0000 !Lower Taper-radius

10.0000 !Upper Taper-radius

2.8793 !Not used

33.8667 !Valency undercoordination

1.8641 !Valency angle/lone pair parameter

1.0563 !Valency angle

2.0384 !Valency angle parameter 
$6.1431 !$ Not used

6.9290 !Double bond/angle parameter

0.0283 !Double bond/angle parameter: overcoord

0.0570 !Double bond/angle parameter: overcoord

-2.4837 !Not used

5.8374 !Torsion/BO parameter

10.0000 !Torsion overcoordination

1.8820 !Torsion overcoordination

-1.2327 !Conjugation 0 (not used)

2.1861 !Conjugation

1.6253 !vdWaals shielding

0.0100 !Cutoff for bond order $\left({ }^{*} 100\right)$

4.8414 !Valency angle conjugation parameter

3.5857 !Overcoordination parameter

38.6472 !Overcoordination parameter

2.1533 !Valency/lone pair parameter

$0.5000 !$ Not used

$1.0000 !$ Not used

5.0000 !Molecular energy (not used)

0.0000 !Molecular energy (not used)

6.7716 !Valency angle conjugation parameter

7 ! Nr of atoms; cov.r; valency;a.m;Rvdw;Evdw;gammaEEM;cov.r2;\#

alfa;gammavdW;valency;Eunder;Eover;chiEEM;etaEEM;n.u.

cov r3;Elp;Heat inc.;n.u.;n.u.;n.u.;n.u.

ov/un;val1;n.u.;val3,vval4

C $1.3742 \quad 4.0000 \quad 12.0000 \quad 1.9684 \quad 0.1723 \quad 0.8712 \quad 1.2385 \quad 4.0000$

$8.7696100 .0000 \quad 4.000031 .082379 .5548 \quad 5.7254 \quad 6.9235 \quad 0.0000$

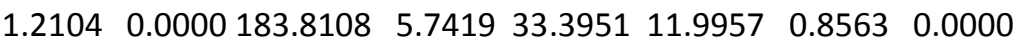

$\begin{array}{llllllll}-2.8983 & 4.7820 & 1.0564 & 4.0000 & 2.9663 & 1.6737 & 0.1421 & 14.0707\end{array}$

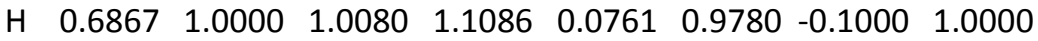

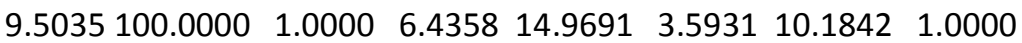




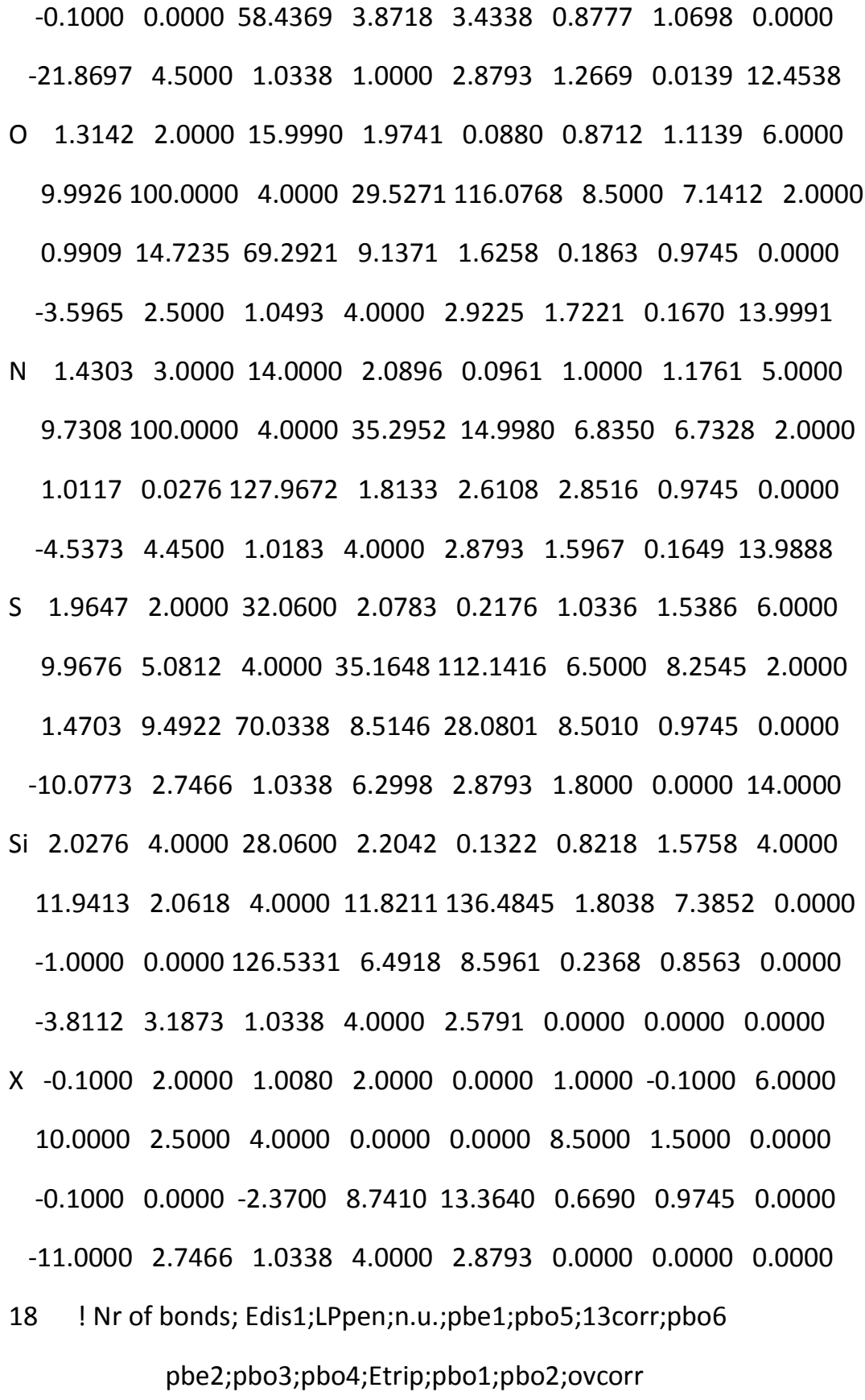




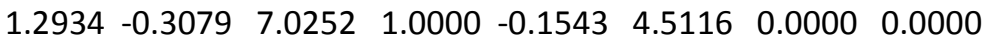

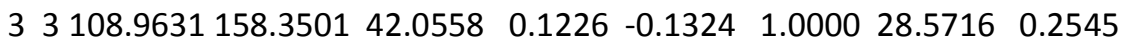

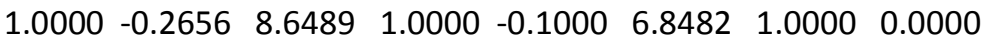

$14128.9104171 .2945100 .5836-0.1306-0.4948 \quad 1.000026 .7458 \quad 0.4489$

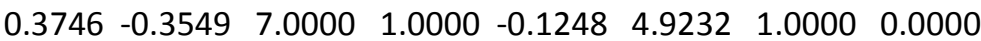

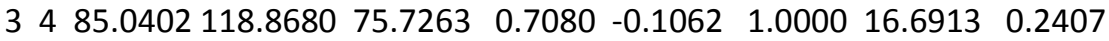

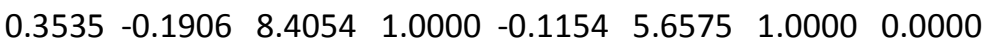

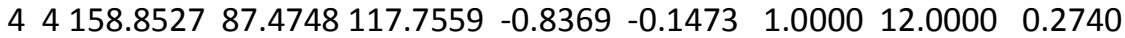

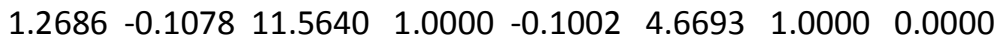

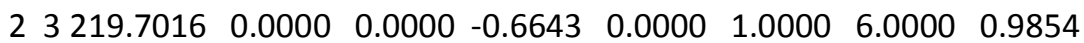

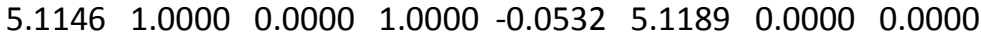

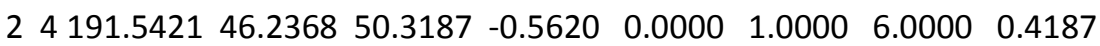

$\begin{array}{llllllll}6.7588 & 1.0000 & 0.0000 & 1.0000 & -0.0438 & 5.9161 & 0.0000 & 0.0000\end{array}$

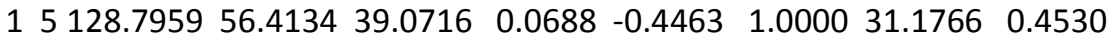

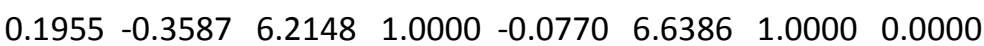

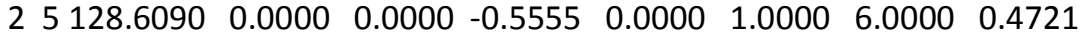

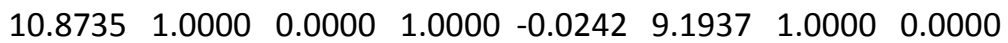

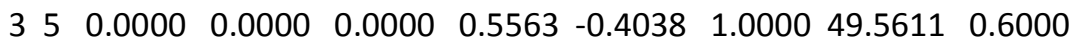

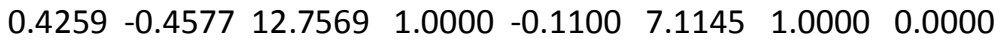

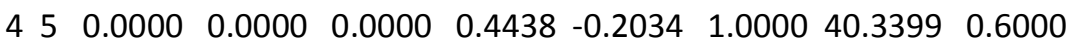

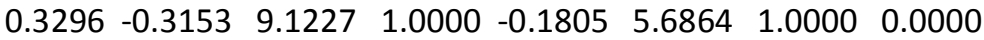

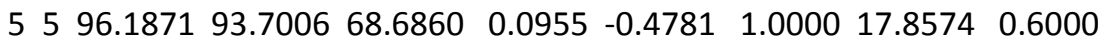

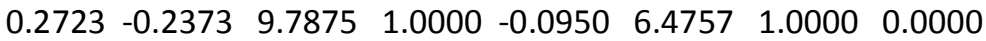

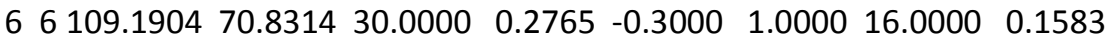

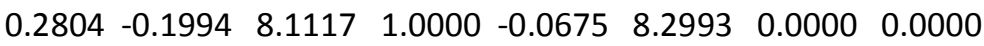

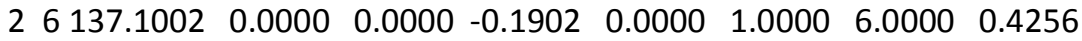

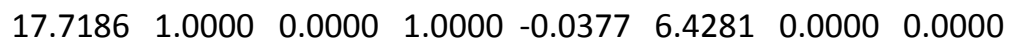

$36191.174352 .073343 .3991-0.2584 \quad-0.3000 \quad 1.000036 .0000 \quad 0.8764$

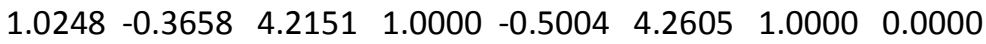

10 ! Nr of off-diagonal terms; Ediss;Ro;gamma;rsigma;rpi;rpi2

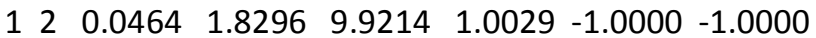




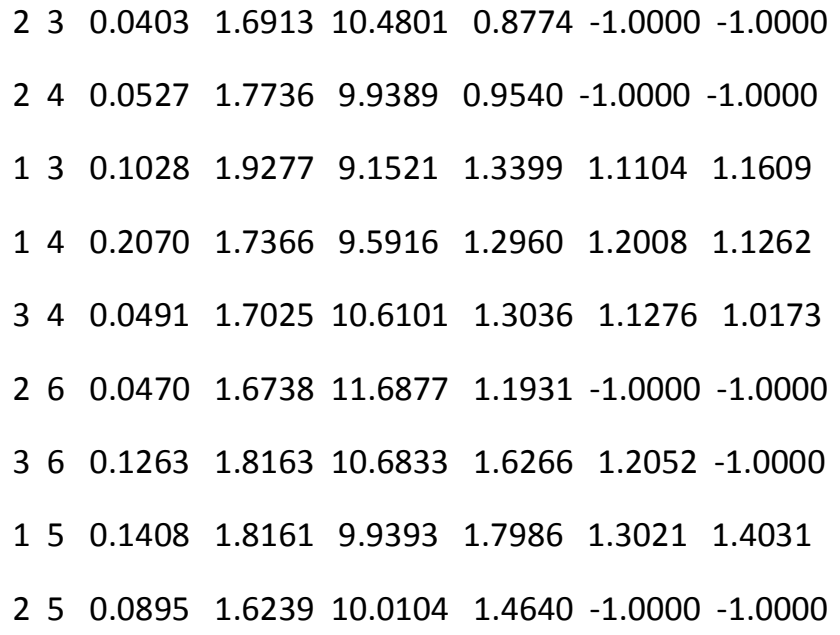




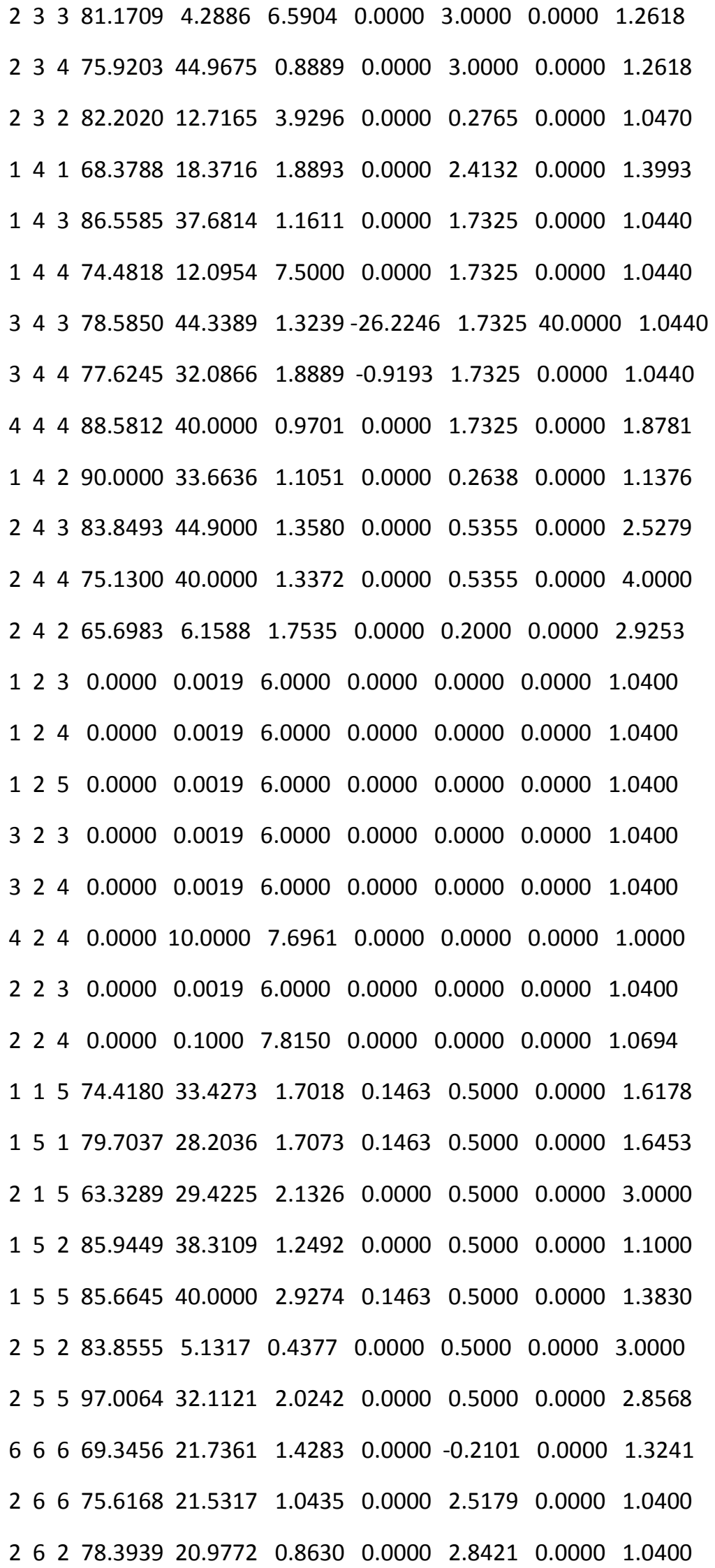




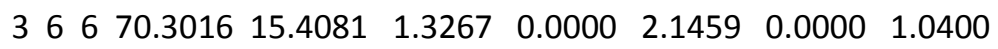

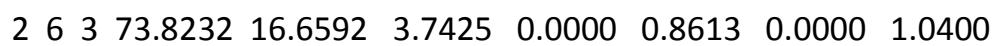

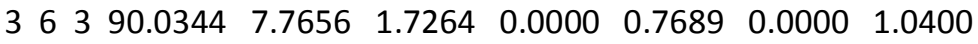

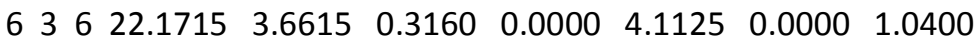

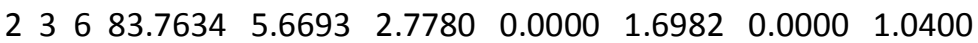

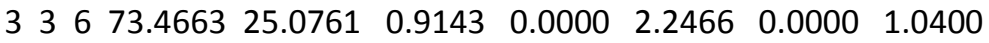

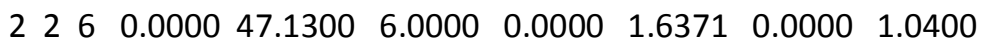

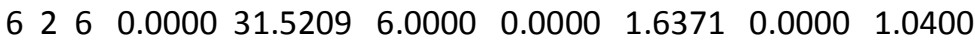

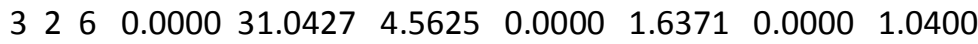

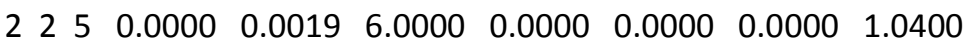

31 ! Nr of torsions;at1;at2;at3;at4;;V1;V2;V3;V2(BO);vconj;n.u;n

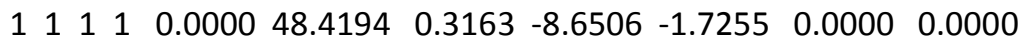

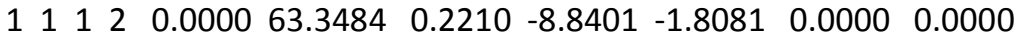

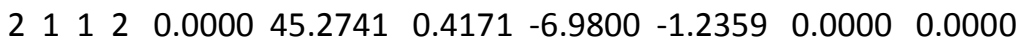

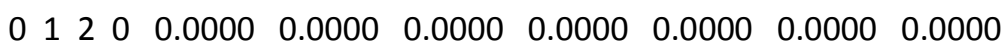

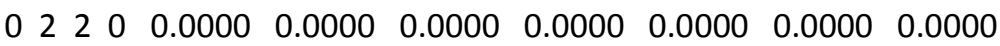

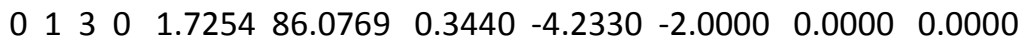

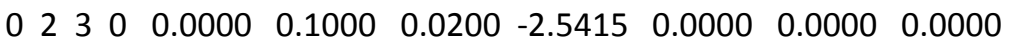

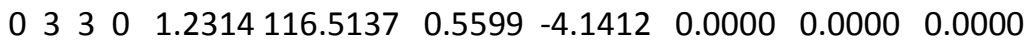

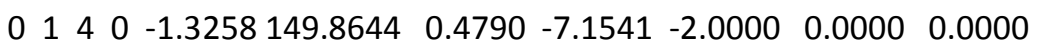

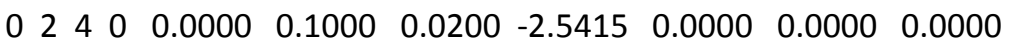

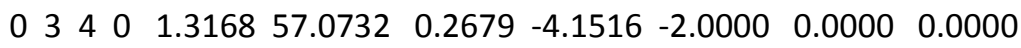

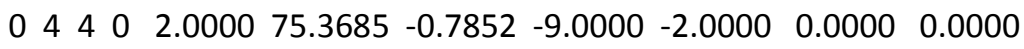

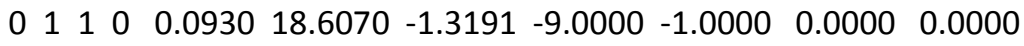

$4144 \quad-2.000020 .6655-1.5000-9.0000-2.0000 \quad 0.0000 \quad 0.0000$

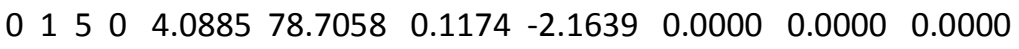

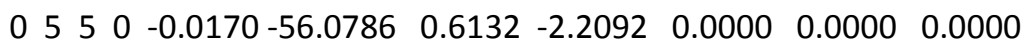

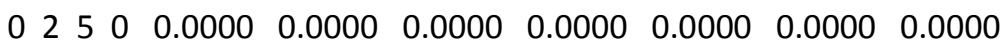

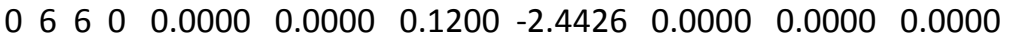

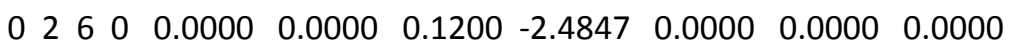

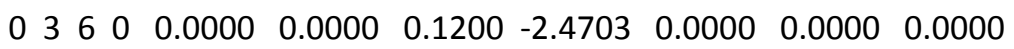




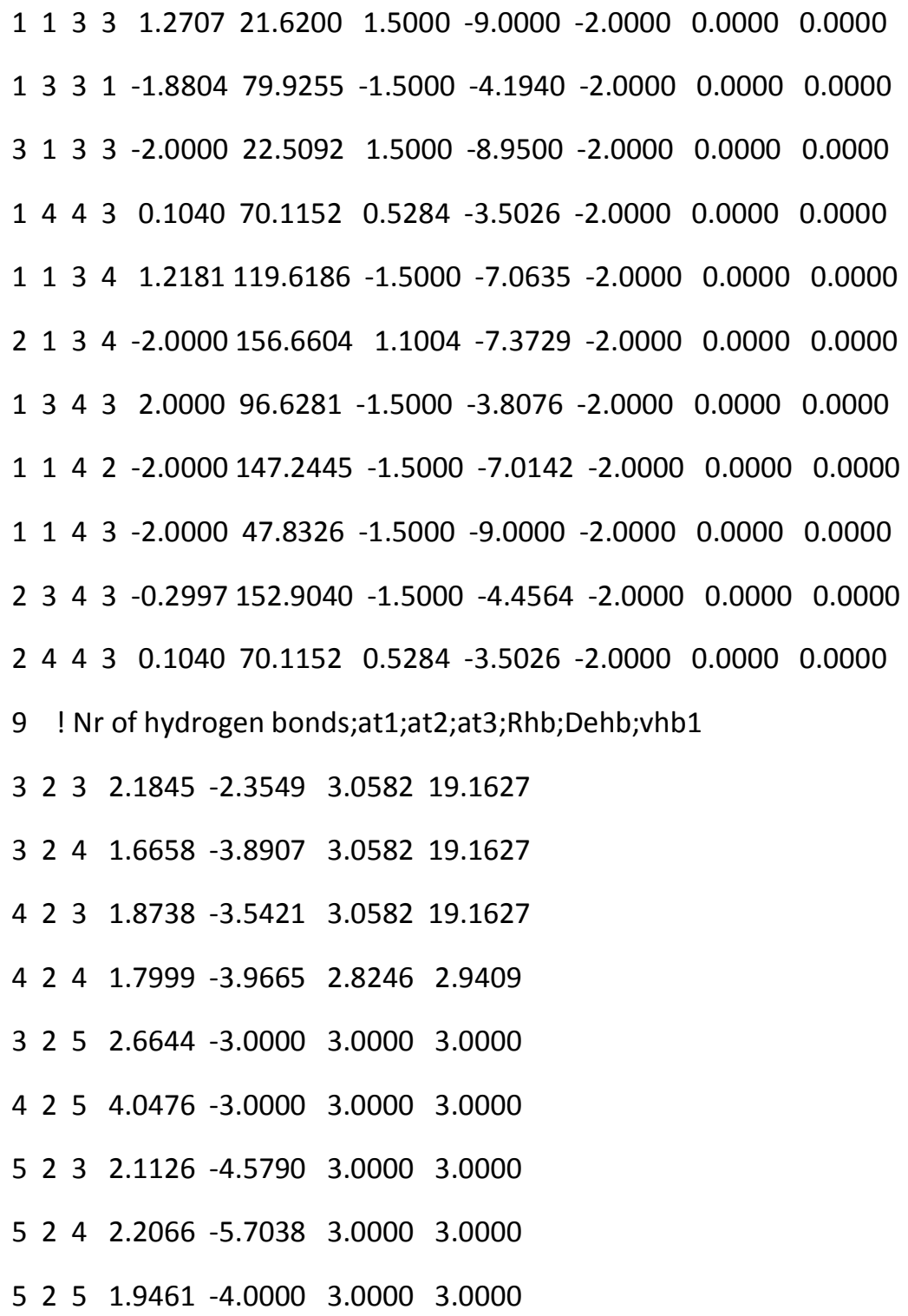

